\title{
Design Considerations to Tailor Unmanned Aerial Vehicles for Martian Geoclimatic Conditions
}

\author{
J. C. Johnson ${ }^{1,2,3}$, P. A. Johnson ${ }^{1,3}$, A. A. Mardon²,3 \\ ${ }^{1}$ Faculty of Engineering, University of Alberta. \\ ${ }^{2}$ Faculty of Medicine and Dentistry, University of Alberta. \\ ${ }^{3}$ Antarctic Institute of Canada. \\ jcj2@ualberta.ca; paj1@ualberta.ca; aamardon@yahoo.ca
}

\section{INTRODUCTION}

Unmanned aerial vehicles (UAVs) hold tremendous promise for the collection of atmospheric measurements, soil sampling, exploring lava tubes, and the search for extant life on Mars. It is important to consider and describe some specifications before deployment for design of UAVs that consider the martian geography, ubiquitous dust devils, and climatology.

\section{Power Considerations}

The craft must be capable of working in low-light, no-atmosphere environment and possess either a relatively long rotor battery life or some alternative form of fuel. For example, NASA's Extreme Access Flyers are using jets of oxygen gas and water vapor as propellant to aerially maneuver and are estimated to last 20 minutes [1].

It should also be built with a mechanism to recharge by autonomously returning to the mothership and/ or conservatory mechanisms such as use of lightweight fiberglass material. Fuel increase capacity can be problematic as increasing weight capacities for fuel come at the cost of aerodynamic endurance.

Another application is the use of solar-powered UAVs for over grounds exploration. This is an interesting fuel source to keep in mind as it offers a more elegant solution to the limited power supply problem with a hybrid of batteries, photovoltaic cells and regenerative fuel cells optionally. Unfortunately, solar power is a double-edged sword and becomes a limitation in more prevalent low-light conditions.

\section{High Climb Rate and Loiter Speed}

Some crater walls can have tilts greater than 30 degrees, which prevent a standard rover from being able to traverse. Higher ranges of operating altitudes are more desirable as they improve chances of survival and coverage. It is important to ensure the loiter speed is also relatively high so there is higher payload coverage that is, area for sensors to scan. [1]

\section{Data-Link Bandwidth Capabilities}

Interfacing with mission planning, control, and monitoring will require optimization with satellites and hubs. There should also be a system functionality for controlling the flight route plan, payload plan, and a direct connection to the ground control station.

The bandwidth is also a function of the payload, meaning the more sensor and motor technology incorporated, the poorer the output quality. This is why the relative cost-benefit ratio of each sensor added should be carefully considered to assess compliance with the mission goals. 
Design Considerations to Tailor Unmanned Aerial Vehicles for Martian Geoclimatic Conditions

Navigation Devoid of Global Positioning Systems (GPS)

UAV flight data is typically geo-referenced in Earth by GPS satellite data, however this is a challenge for tracking heading information in Mars. A combination of real-time 3D mapping, solar positioning, and radio or optical beacons produced by other UAVs may serve to landmark and function to ease the task wayfinding. In this manner, UAVs can serve as a pseudo- GPS for future missions.

\section{Rotors in Mars Gravitational Field}

Ramsley and Head suggest that power-to-lift efficiencies of UAV rotors drop from $\sim 80 \%$ in terrestrial conditions to $\sim 14 \%$. This demonstrates a demand to overcome the gravitational impedance that is applied to the rotor mechanics.

If this rotor efficiency can be optimized to $>30 \%$, it is possible to increase flight duration and improve payload capacities. Proposals to do so include solarpowered wings with rotor propulsion and helicopterinspired designs.

\section{Loss of Contact with Ground Control Station}

Lengthier transmission distances, geographic interference, and spurious losses of contact with the base station are foreseeable events. If there is functionality that ensures the data collected by the relevant sensors are retained, it is possible to recover these measurements when the UAVs return to the line-of-sight of the ground control stations.

\section{CONCLUSION}

The search for extant life on Mars is intimately linked with building a better systems surrounding geographic surveillance and habitat scanning. Designing UAVs for the martian infrastructure with the tradeoffs for payload, bandwidth, maneuverability, and convenience are essential subjects for deliberation and cannot be understated.

\section{REFERENCES}

1. Hassanalian, M., Rice, D., Abdelkefi, A. Evolution of space drones for planetary exploration: A review. Progress in Aerospace Sciences 97, 61-105.

2. Singer C. (2012) Ultralight Solar Powered Hybrid Research Drone. Concepts and Approaches for Mars Exploration, 4059.

3. Ramsley, K.R and Head J.W. (2015) Exploring Mars with Micro-UAV Squadrons and High Data Rate Communications. 46th Lunar and Planetary Science Conference, 1185.

Citation: J. C. Johnson, P. A. Johnson, A. A. Mardon. "Design Considerations to Tailor Unmanned Aerial Vehicles for Martian Geoclimatic Conditions". American Research Journal of Humanities and Social Sciences, vol 5, no. 1, 2019, pp. 1-2.

Copyright (c) 2019 J. C. Johnson, P. A. Johnson, A. A. Mardon, This is an open access article distributed under the Creative Commons Attribution License, which permits unrestricted use, distribution, and reproduction in any medium, provided the original work is properly cited. 